\title{
Effectiveness of mobility management in a transportation policy aimed at achieving the Kyoto Protocol - Kyoto project for studying an efficient car utilization
}

\author{
T. Murao ${ }^{1}$, D. Nakagawa ${ }^{2}$, R. Matsunaka ${ }^{2} \&$ T. Oba ${ }^{2}$ \\ ${ }^{I}$ Department of Policy Planning, Kyoto Prefecture, Japan \\ ${ }^{2}$ Department of Urban Management, Kyoto University, Japan
}

\begin{abstract}
As the first commitment period of the Kyoto Protocol comes to a start, the reduction of greenhouse gas emissions becomes an urgent topic in the transport sector. Although the share of car use in terms of a modal split in Kyoto, where the Kyoto Protocol was born, is rather low compared with other similar scale metropolitan areas around the world, activities concerning all citizens aimed at reducing their car use have become very popular.

In particular, Mobility Management, a transportation policy which controls excessive car use by using communication skills, is producing good results.

In this paper, we will show the successful results of the "Kyoto Project for Studying an Efficient Car Utilization" by introducing two specific projects. One of these is an example of how ridership for railways has dramatically increased as a result of the widespread execution of Workplace Mobility Management, and the other is an example that the ridership of buses has increased because of integration with commuter buses in industrial areas.

Based on these results, and paying attention to the execution process of these projects, we analyze the factors leading to the successful results.

Finally, we propose ideas for use in other areas with similar characteristics.

Keywords: workplace mobility management, achieving the Kyoto Protocol, efficient car utilization, implementation process.
\end{abstract}




\section{Introduction}

The first commitment period of the Kyoto Protocol has already started and achieving the goals laid out in it has become a pressing task. Therefore, Kyoto City and Kyoto Prefecture, the birthplace of The Kyoto Protocol, have individually enacted their own Global Warming Countermeasure Ordinance. In the ordinance, they set the target of a 10 percent reduction in greenhouse gas emissions exceeding the national target of 6 percent, compared with those in 1990.

Since the Kyoto Metropolitan Area is relatively densely populated, the service level of public transportation is relatively high. It is possible to curb that excessive car use, and there remains the condition in which people can live without using cars. According to Newman and Kenworthy [1], the feature of ideal urban areas in the future is centering on the high-density core, the network of railways extends to the suburbs and the urban areas are formed centering on each station. That is very similar to Fig 1, that of Kyoto in respect to the transportation network. In other words, Kyoto is quite possibly the model sustainable urban area.

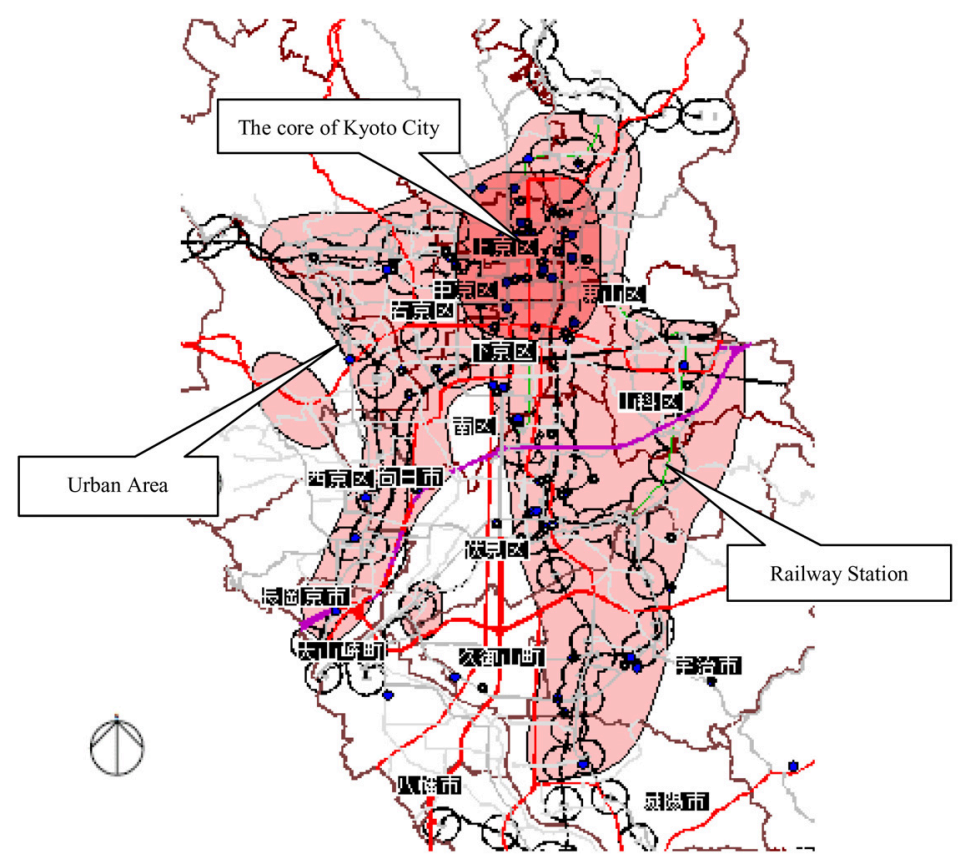

Figure 1: Transportation network in the Kyoto Metropolitan Area.

However, the amount of greenhouse gas emissions tends to increase in Kyoto as well, and the share of cars as a means of transportation is continuously increasing. Therefore, it has become necessary to tighten up the expansion and 
dispersion of the urban area, and, in the meantime, take aim at the pressing task of achieving the goals of the Kyoto Protocol. In order to accomplish this, the prefectural government is addressing the needs by reviewing the individual way of life and changing the public mindset in respect to car dependence.

Among others, Mobility Management (hereinafter referred to as MM), a transportation policy encouraging voluntary change of transportation behavior, has already been implemented on a broad scale through communication between the administration and citizens in comparison with other cities around the world.

In this paper, to begin with, let us introduce the actual implementation of MM in the Kyoto Metropolitan Area. Then, focusing on the practical procedures in the successful cases of MM, we will clarify the factors that led to their success, and at the same time, provide useful information for the implementation of the policy to curb the excess use of cars in other cities.

\section{Reference review}

According to Fujii [2], it was the seminar held by the Japan Society of Civil Engineers in January 2004, that the term "Mobility Management" was used for the first time in public. It was used for the first time in an official document submitted in 2004, the Kinki Council for Regional Transport of the Ministry of Land, Infrastructure, Transport and Tourism. So, the history of this term is short in Japan. There have been many research projects and reports concerning MM.

Taniguchi and Fujii [3] overviewed the process addressing MM in the past in Japan, and pointed out that it is important that MM shifts from the experimental and research stage to the more practical and political stage. As for some actual example studies from the Kyoto Metropolitan Area, there have been both an experimental study targeting commuters in Uji City by Hagihara et al. [4], and an actual example of the bus system introduced around the industrial park cooperatively run by companies of the industrial park as part of the application using MM by Sakai et al. [5].

This paper points out the factors that led to success in the MM project, clarifies the significance of these achievements from the practical viewpoint of paying attention to the implementation process of each policy.

\section{Outline of mobility management in the Kyoto metro area}

The population of the Kyoto Metropolitan Area is 2.30 million as of 2000 (number of persons engaged: 1.07 million), and its total area is $1,957 \mathrm{~km}^{2}$. It is a region containing both cities and towns as shown in Fig.2, belonging to the Kyoto Prefecture that are strongly integrated socially and economically with the core of Kyoto City.

The share of cars as a means of transportation in the Kyoto Metropolitan Area, as shown in Table 1, is relatively low compared with other cities around the world roughly the same size. However, the usage of cars tends to increase in suburban area, in particular, so the prefectural government is dealing with measures to reduce the share of cars. 
Table 1: Transition of the share of cars as a means of transportation in the Kyoto metro area [6].

\begin{tabular}{|c|c|c|c|c|c|}
\hline Year & $\begin{array}{c}\text { Railway } \\
(\mathbf{\%})\end{array}$ & $\begin{array}{c}\text { Bus } \\
(\%)\end{array}$ & $\begin{array}{c}\text { Car } \\
(\mathbf{\%})\end{array}$ & $\begin{array}{c}\text { Two- } \\
\text { wheeler }\end{array}$ & $\begin{array}{c}\text { Walk } \\
(\mathbf{\%})\end{array}$ \\
\hline 1980 & 12.9 & 8.3 & 22.8 & 19.8 & 36.3 \\
\hline 1990 & 14.8 & 5.6 & 27.8 & 22.7 & 29.0 \\
\hline 2000 & 15.6 & 4.6 & 32.4 & 24.0 & 23.4 \\
\hline
\end{tabular}

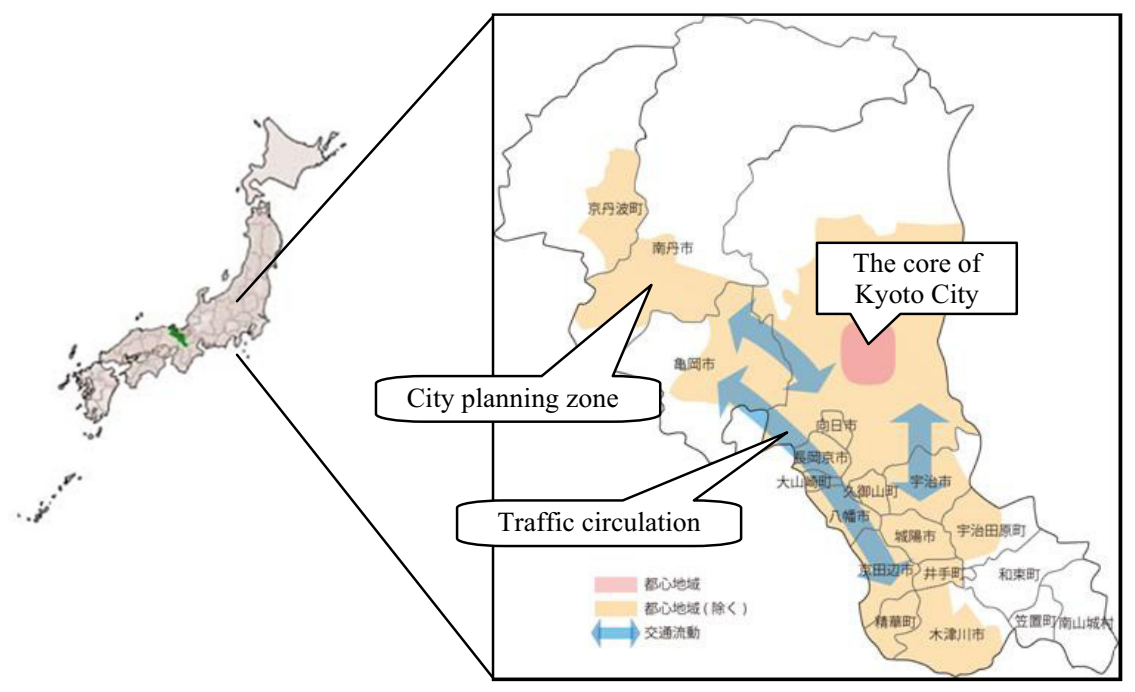

Figure 2: $\quad$ Kyoto Metropolitan Area.

The government of Kyoto Prefecture has fully implemented the policy since 2004 and developed a measure called the "Kyoto Project for Studying an Efficient Car Utilization" as a national model project based upon "the Basic Plan for the Traffic Demand Management by Kyoto Prefecture".

This plan, in particular, is aimed at realizing its goal by involving as many citizens as possible based on MM as the core of the measures is communication between the citizens and local government.

The MMs targeting residents, the one targeting commuters cooperating with their company and the one targeting students and their families etc. is improved mainly in the areas where the issues of transportation become obvious as national model project.

As a result, in 2007, the measures for commuters targeting approximately 9,000 people in five cities and towns including Kyoto City, Uji City and Kizugawa City, the measures for residents targeting 133,000 households in eight cities and towns, and the measures for elementary school students targeting approximately 9,000 in two cities and a town were implemented. Furthermore, as for renewing their license every five years, all licensed drivers are provided with 
the information to motivate them to practice eco-driving and change to public transportation from the use of their own private car. The number of targeted licensed drivers is over 220,000 a year. In addition, this kind of information is provided through local newspapers (500,000 copies) and FM radio. The Ministry of Land, Infrastructure, Transport and Tourism, Kyoto Prefectural Government and Kyoto Municipal Government have established the Kyoto Metropolitan Area MM Council, and they are developing these activities with the participation of all citizens, being guided by specialists.

The following are two examples of measures for commuters (MM targeting workplaces):

Generally speaking, it is not easy to curb private car use for commuting, because private car use is selected by commuters as the most appropriate method for getting to where they want to go from the viewpoint of cost, time, and convenience etc. Therefore, it requires coordinated efforts involving companies and workers groups that are provided commuter allowances.

The result of the questionnaire administered to companies shows that, even in the areas in the neighborhood of railway stations, many people are using private cars, and that unsatisfactory connection between railway stations and the bus system causes people to commute by car.

There are two examples of how to respond to such problems:

\section{(1) Uji district commuter traffic pilot program}

Although there are three railways lines, JR, Keihan and Kintetsu in Uji City, and bus lines are relatively well developed, the percentage of automobile commuters is high. Therefore, one-shot TFP, a part of MM, was implemented targeting commuters (approximately 4,400) in September 2005 with the cooperation of the Uji Chamber of Commerce and companies. Also in 2006, the same communication questionnaire was conducted again.

One-shot TFP is a one-time communication questionnaire conducted targeting commuters. This questionnaire is attached to a map carrying the summary of public transportation for commuters and a booklet for the purpose of motivating the use of public transportation, and ask respondents to answer

(from 7 o'clock to 9 o'clock) excluding commuters pass holders

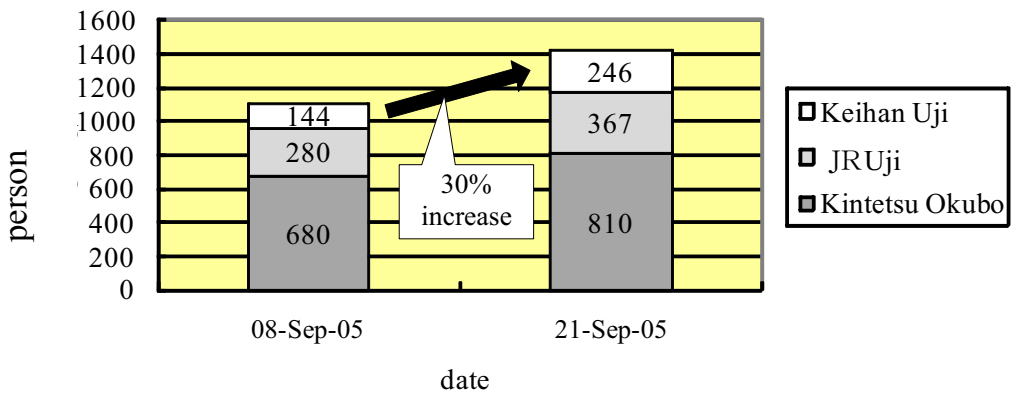

Figure 3: $\quad$ Change in the number of passengers getting off (2005). 
(from 7 o'clock to 9 o'clock) excluding commuters pass holders

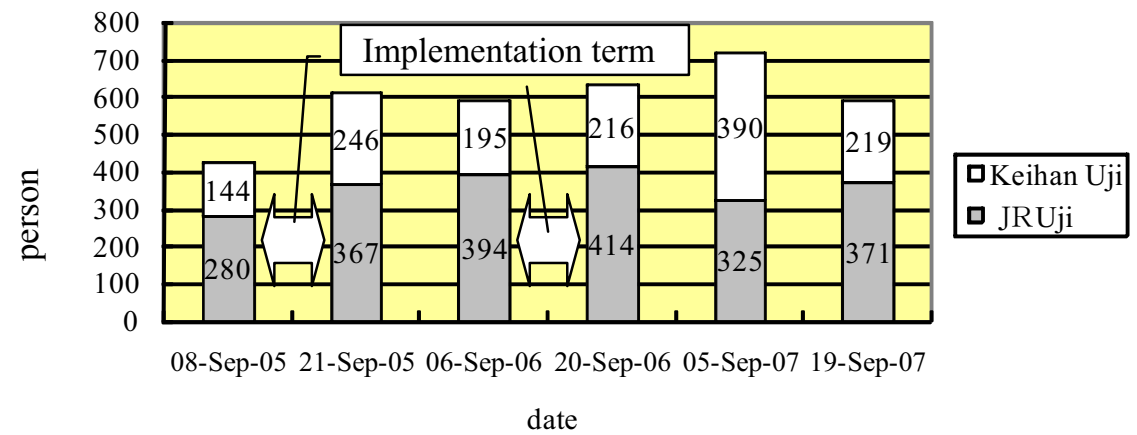

Figure 4: Change in the number of passengers getting off during commuting hours (2005-2007).

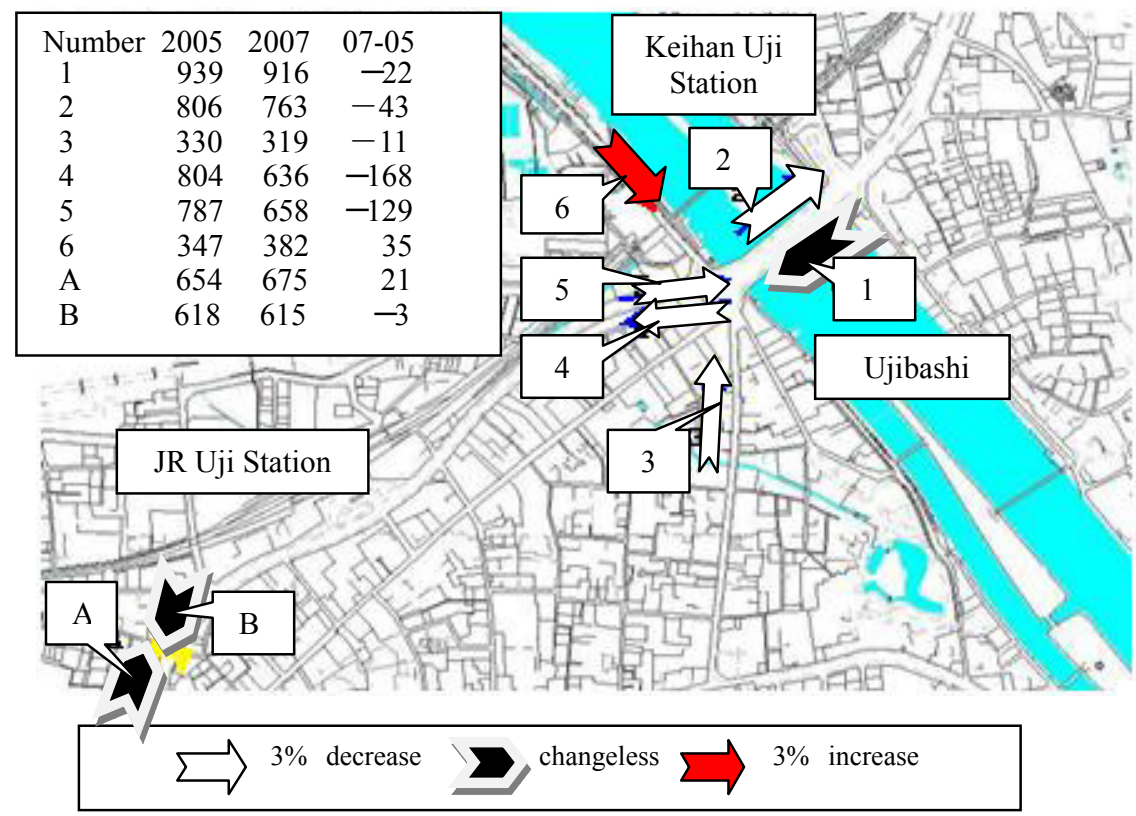

Figure 5: Change of the car traffic volume in the Uji area (during commuting hours).

the questions and confirm the answers in the order of: 1) everyday travel behavior, 2) how to commute in ways other than a car, 3) what time is required to arrive at the station, and 4) what are the merits for commuting in ways other than a car.

The amounts of traffic were surveyed after the implementation of the questionnaire, and as a result, the users of the three railway stations (JR Uji station, Keihan Uji station and Kintetsu Okubo station) excluding commuters 
pass holders for use during the morning commute increased approximately $30 \%$, as shown in Fig.3, and it was confirmed that the amount of car traffic around workplaces decreased, and pedestrians and bicyclists increased.

As a result of the surveys of the traffic amounts performed in 2006 and 2007, the conditions of railway users and car traffic volume around Ujibashi after the 2005 survey have been maintained and it was confirmed that the effects of these measures have continued as shown in Fig.4, 5.

Several secondary effects can be noticed through this measure. For one, the opinions of commuters were heard through the one-shot TFP, and the administration, companies and railway operators could share these opinions for their future tasks.

As for another, because the effects of the measures of the first year were apparent, railway operators recognized the effects of measures of MM, and established a confidential and cooperative relationship with the administration.

From the above, the experiment in this area had great momentum in other areas of Kyoto Prefecture.

\section{(2) Approach at Kuze industrial park}

Meanwhile, if the service level of public transportation is not high enough for commuting, the provision of the information by MM could cause adverse effects, so that it is necessary to improve the level of public transportation between railway stations and workplaces. Because there was a recognition that traffic problems affected business activities among business people in Minami Ward and Fushimi Ward in Kyoto City, the group of them established a workshop together with the Kyoto Chamber of Commerce and the administration, and launched a pilot program of cooperative shuttle bus service for one month from November 2006.

At the same time, they urged commuters to utilize the shuttle bus, by implementing a communication questionnaire, publishing a newsletter, providing

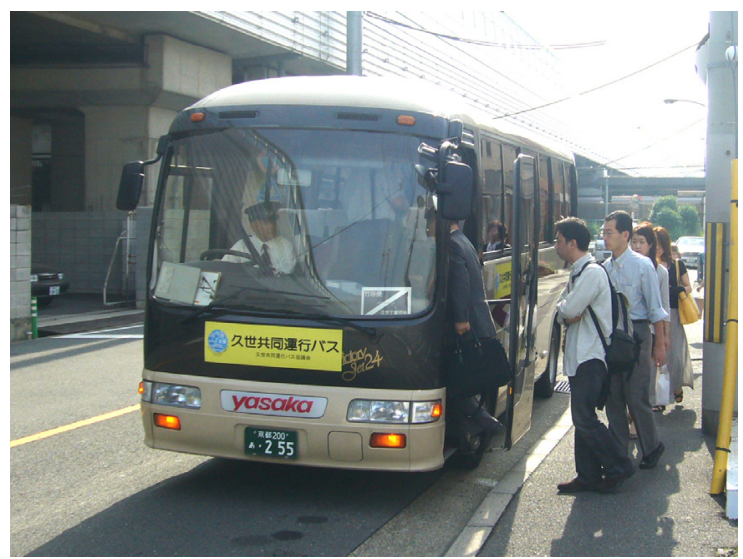

Figure 6: The current situation of shuttle bus. 
commuting plans using the shuttle bus service, holding workshops etc., and thus provided communication to each commuter.

As a result, shuttle bus users increased 1.7 times during the pilot program, and since May 2007, real service has been provided and 22 companies are running bus service.

\section{Discussion on success factors}

The workplace MM in Uji and Kuze industrial parks could bring about great success. High participation rates show that it has been successful at first. The recovery rates of questionnaires for commuters were extremely high such as $68.8 \%$ and $59.9 \%$ in the Uji projects of 2005 and 2006 , respectively, and $41.8 \%$ in the Kuze project. It was important that many participants could be recruited. Here are the following reasons as to why this was obtained:

\section{(1) Conformity between MM project and needs of companies}

In addition to the awareness that traffic jams during commuting hours and the access to railway stations are shared problems in the community as a whole, the approach to the workplace MM was combined with the companies' measures against global warming and health care measures for employees, and the momentum of companies to cooperate in curbing private car use for commuting, so that it became possible to perform the questionnaires as a part of a company's business.

\section{(2) Large scale and individual correspondence}

It brought about great success with the contents and design of the commuting map being changed, and administrative officers could visit companies to explain the workplace $\mathrm{MM}$ in detail and obtain cooperation, and distribute and collect the questionnaires.

\section{(3) Dissemination of the improvement progressed by the project}

As a result of urging railway operators and administration with taking action by conveying the voices of users participating in the project in 2005, several enhancements including the improvements of the timetable of railways, sidewalks and street lights etc. proceeded considerably. More over voice of users were fed back to the participants, so that they were convinced of the significance that they voluntarily engaged in these projects. These things became the factors that led to the success of this project.

\section{(4) Organizational structure allowing for the continuation of the project}

The parties concerned in commuting and traffic range from various fields and they had to be discrete about approaching this project. However, led by Kyoto Prefecture Government, an organizational structure was established to share the purpose of this project and continuously work on this project, supported by the experts in various fields, and as a result, communication among the parties concerned was effective. 


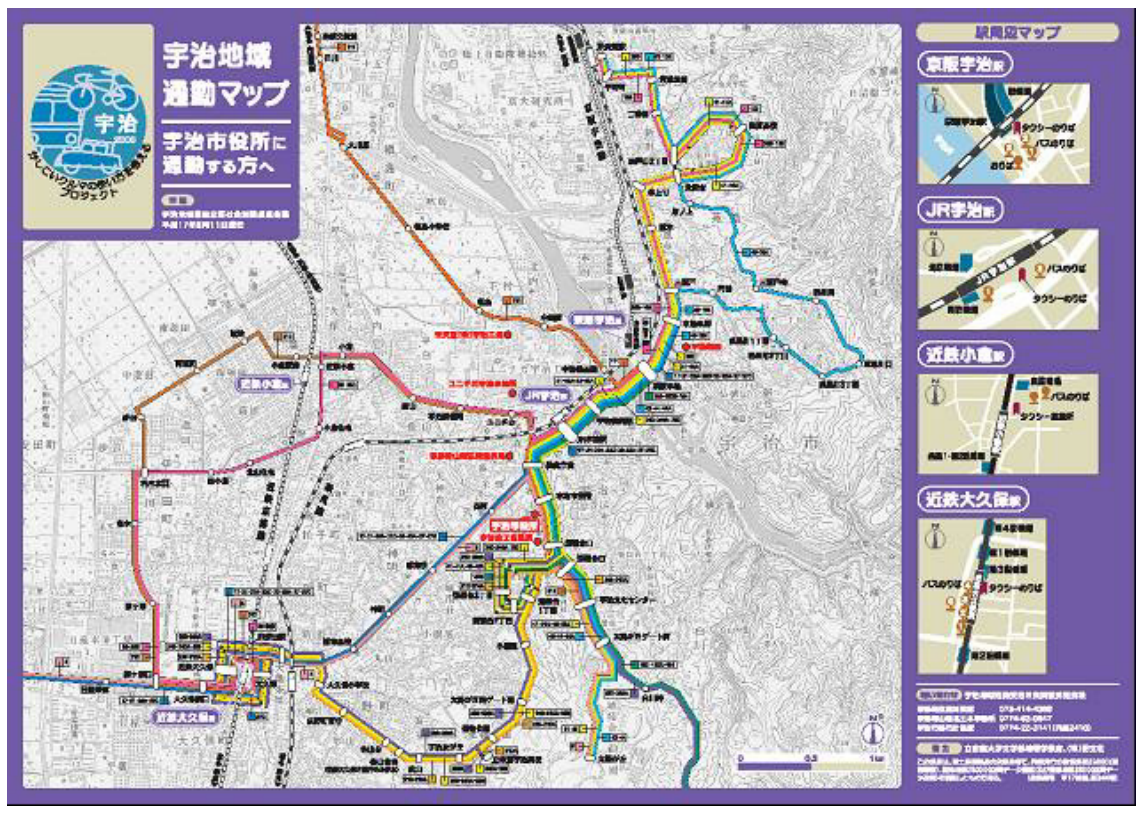

Figure 7: Uji district commuting map.

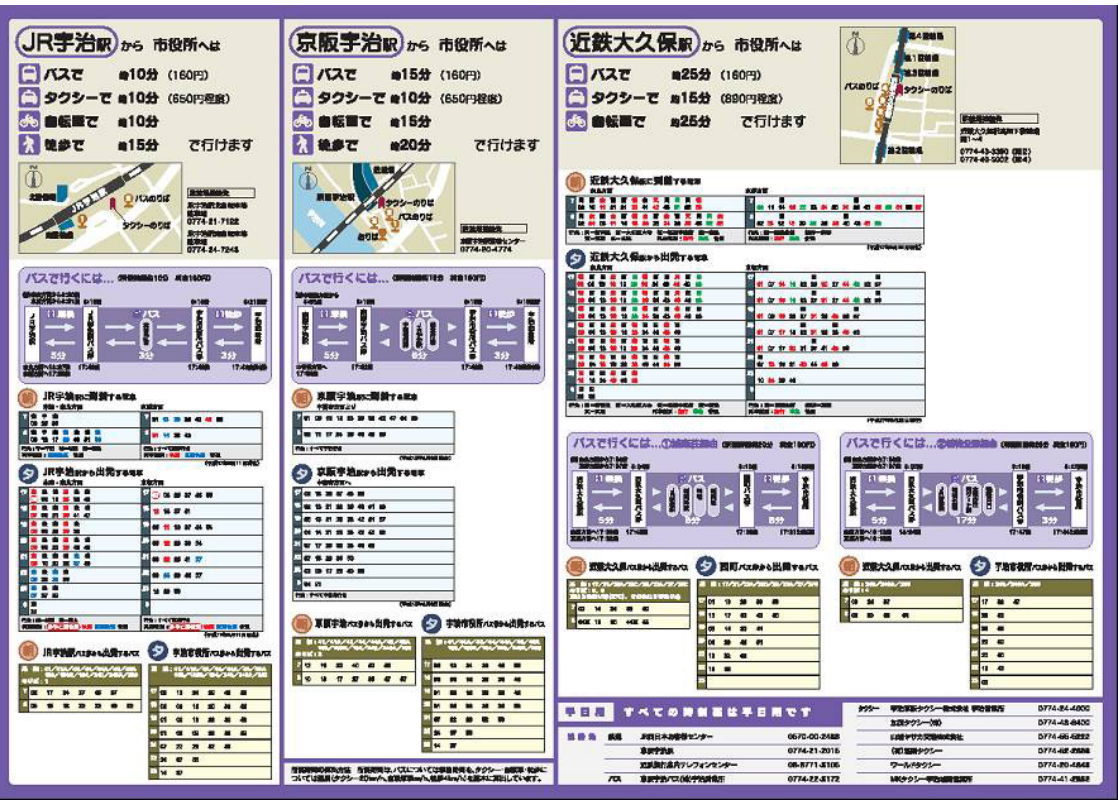

Figure 8: $\quad$ Uji district commuting timetable. 
Many other pilot programs have disappeared after the completion of the experiment in Japan. However, it depends upon whether the purpose of the project is clarified and the framework and consensus to continue the program can be made during the period of the experiment.

\section{Conclusion}

Transport operators are run by private enterprises independent from the administration and the parties concerned range from various fields in Japan. However, public transportation is based upon a complementary relationship among these parties as a network, so that the parties concerned have to counter car use behavior as a team, otherwise, the shift from private car use to public transportation use cannot proceed.

We would like to point out that organizing a vigorous transportation network resembles organizing a strong sports team in various points as follows:

\section{(1) Credible strategy and retention}

It is necessary to know one's own characteristics (strong points, weak points), and share a vision based on them and plan a strategy to realize that vision.

\section{(2) Existence of a good leader}

In each district where remarkable transportation measures have been realized in the world, the existence of the top exercising strong leadership can always be confirmed. Since transportation policy is an approach to changing society, it has immeasurable effects; therefore, it requires a strong will. The existence of a strong leader is seen in the realization of LRT in Toyama, Portland and Strasbourg, Road Pricing in London and the bus system in Seoul.

\section{(3) Teamwork}

Movement by public transportation should be considered from the departure point to the destination as a whole. Any bad railway connection makes the public transportation system useless. Therefore, teamwork among public transport, such as coordination among transportation, installation of bicycle parking areas in front of the station etc. is very important.

\section{(4) High physical ability of each player}

In order to counter comfortable transportation by car, it is required to make stations and trains comfortable and easy to use.

\section{(5) Supporters}

It is important for the public transportation system that supported by users and draws various opinions including complaints from them in a similar way that a strong sports team has strong supporters. 


\section{(6) Enhanced support system}

Since the public transportation system is social facility, it is important that the administration, NPO etc. support it by providing guidance and knowledge, utilizing the government subsidy system and the national model business, holding a council of advisors etc.

With the comprehensive development of the above six points, the public transportation system can work effectively.

\section{References}

[1] P.Newman and J.Kenworthy, "Sustainability and cities" .Island Press, pp185, Fig.4.3, 1999

[2] Satoshi Fujii, "Development of Mobility Management in Japan" IATSS Review Vol.31, No.4, 2006

[3] Ayako Taniguchi, Satoshi Fujii "Current situation of soft measures for car use reduction in United Kingdom" Planning Study, Collection of Paper Infrastructure Planning Review 23,(4). pp981-988, 2006

[4] Go Hagihara, Toshimichi Murao, Kazuyuki Shimada, Keiko Yosiura, and Satoshi Fujii "Aggregative effects of large scale workplace mobility management and comparative analyses on effects of mobility management measures" Journal of Infrastructure Planning and Management, Vol. 64, No. 1, pp.86-97, 2008 .

[5] Hiromu Sakai, Satoshi Fujii, Toshimichi Murao "A management work to introduce a jointly-operated bus system by multiple companies workplace mobility management based on diverse types of communication in a transportation poor area" Infrastructure Planning Review, 25,(4), pp10171024,2008

[6] Keihanshin Urban Transport Planning Conference "The Fourth Keihanshin Urban Area Person Trip Survey Report, Master File Summary Version" 2002 